\title{
Feedback control with finite accuracy: more knowledge and better control for free
}

\author{
Joseph D. Skufca ${ }^{\mathrm{a}, *}$, Erik M. Bollt ${ }^{\mathrm{b}}$ \\ a Department of Mathematics, United States Naval Academy, Annapolis, MD, USA \\ ${ }^{\mathrm{b}}$ Department of Mathematics and Computer Science, Clarkson University, Potsdam, NY 13699-5815, USA
}

Received 19 April 2002; received in revised form 20 October 2002; accepted 3 January 2003

Communicated by E. Kostelich

\begin{abstract}
It is known [Phys. Rev. Lett. 63 (11) (1990) 1196-1199] that a system can be controlled near an unstable period- $k$ point by applying a small perturbation signal to some parameter in the system. The required perturbation is calculated as a function of the current system state. We consider that, in applications, such stabilizing algorithms can be implemented with only finite accuracy. The error associated with the finite accuracy will grow exponentially, requiring repeated application of the parameter perturbation to keep the system near the fixed point. We show that under a repeated perturbation control algorithm, the resultant dynamical system is a piecewise expanding map that is well approximated by a Renyí transformation. Additionally, we show that by an analysis of the kneading sequences, the system state can be known with greater accuracy than is measured. Furthermore, we modify the standard parametric control algorithm to provide better time averaged control of the system without increasing the complexity of implementation. We demonstrate application of these principles to the 2D case by considering a saddle fixed point of the Ikeda map and the OGY [Phys. Rev. Lett. 63 (11) (1990) 1196-1199] algorithm.
\end{abstract}

(C) 2003 Elsevier Science B.V. All rights reserved.

PACS: 05.45.-a; 05.45.Ac; 05.45.Gg; 02.30.Yy; 47.52.+j; 47.53.+n; 95.10.Fh

Keywords: Control of chaos; Finite measurement control; Finite accuracy measurement; Accuracy improvement algorithm; Kneading theory; Symbol dynamics; Invariant measure

\section{Introduction}

An essential part of a control scheme to stabilize a system is to measure the system and then inject the calculated control signal. Any such control scheme is obviously limited by the accuracy of the measurement system. Starting with 1D maps and the Shinbrot algorithm [2], which was developed to target unstable periodic orbits on a chaotic set, we develop a technique that allows knowledge of the system that is far more precise than the physical measurement system we are using for control. As a separate application of the analysis, we propose an alternative control scheme that requires no additional computations or measurement but provides significantly improved average performance.

* Corresponding author. Tel.: +1-410-293-6749; fax: +1-410-293-4883.

E-mail address: skufca@usna.edu (J.D. Skufca). 
We then apply the general approach to stabilize a saddle in $\mathbf{R}^{2}$ with the OGY algorithm and illustrate that increased knowledge of state and improved control can be achieved simultaneously. We develop our analysis rigorously for the $1 \mathrm{D}$ maps, and we indicate only by numerical examples how the techniques of analysis should be extended to two dimensions.

An essential part of our specification technique relies on using hyperbolicity to our advantage. In that sense, one might be reminded of the data cleaning techniques, such as those of Farmer and Sidorowich [3,4]; however, our methods are substantially different. We introduce a novel application of symbolic dynamics relative to a nongenerating partition, and this turns out to be an essential feature for our application.

\section{Reduction of controlled systems with finite rounding}

\subsection{Background: targeting}

We begin by developing a notation to summarize the targeting algorithm of Shinbrot et al. [2], which quickly steers trajectories to unstable periodic orbits. Beginning with the simplest case: consider a discrete time, 1D system, which is described by

$$
x_{n+1}=f\left(x_{n}\right) .
$$

Assume that the system has an unstable period- $k$ point at $x=p$, such that $f^{k}(p)=p$ and $\mathrm{d} /\left.\mathrm{d} x\left(f^{k}(x)\right)\right|_{x=p}>1$. In general, such a system can be controlled by a feedback control of a parameter associated with the function $f$. Suppose there exists a function $F(a, x)$ and a parameter value $a_{0}$ such that

$$
f(x) \equiv F\left(a_{0}, x\right)
$$

with $F \in C^{1}$ on a neighborhood of $\left(a_{0}, x\right)$. By the implicit function theorem, there exists a continuous function $a(x)$ such that $a(p)=a_{0}$ and

$$
f^{k-1} \circ F(a(x), x)=p, \quad \forall x \in I,
$$

where $I$ is an interval containing $p$. Therefore, for any system state $x$ (sufficiently close to $p$ ), we can calculate a required parameter value $a(x)$ to ensure that the system maps to $p$ after $k$ iterations.

Example. Consider the logistic map with parameter value $a_{0}=4$, so that $F\left(a_{0}, x\right)=a_{0} x(1-x)=4 x(1-x)=$ $f(x)$. This map has a fixed point at $p=0.75$. Then

$$
a(x)=\frac{0.75}{x(1-x)}
$$

and

$$
F(a(x), x)=\frac{0.75}{x(1-x)} x(1-x)=0.75, \quad \forall x \in(0,1) .
$$

For the ideal system, the perturbation of the parameter corrects the system exactly to the fixed point. Only one control perturbation would be required, and the system would remain stabilized for all following iterations. But in a real system, small errors will always persist (due to noise, measurement error, control execution error, etc., even a computer simulation will have roundoff error). If no further action is taken, those small errors will grow exponentially, and the system will eventually be pushed far from the target point. Therefore, to stabilize a real system, repeated application of control will be required. 


\subsection{Generalized analysis, finite rounding}

Consider a dynamical system with an unstable period- $k$ point. Our goal is to stabilize the period- $k$ orbit by varying a control parameter, implementing control once per period, with the additional constraint that the algorithm is implemented with finite accuracy. Specifically, we choose the simple case where the measurement of the system state is of finite accuracy, such that there are only a finite number of possible measured states to describe a continuum of actual states. (A physical example might involve temperature as a state variable, measured with a digital thermometer.) The control algorithm must calculate a parameter perturbation based on the measured state of the system. The new control iteration is given by $F\left(a\left(x_{M}\right), x\right)$, where $x_{M}$ is the measured state of the system. Define an explicit description of the measured state $x_{\mathrm{M}}$, such as

$$
x_{\mathrm{M}} \equiv \frac{\lfloor M x+0.5\rfloor}{M},
$$

which is simply a precise description of "rounding" the measured state to the nearest $1 / M$, where $M$ is a positive real number (typically a large integer). ${ }^{1}$ We define calculated control as

$$
a_{\mathrm{M}}(x) \equiv a\left(x_{\mathrm{M}}\right)
$$

and the finite controlled system by

$$
g_{M}(x)=f^{k-1} \circ F\left(a_{M}(x), x\right),
$$

which is one perturbed iteration followed by $k-1$ iterations of the unperturbed map. Since the "rounding" process is deterministic, this new map is a family of dynamical systems, parameterized by the value of $M$. Our goal is to understand the characteristics of these families.

Example. Again, consider the logistic map, with parameter value of $a_{0}=3.9$. Suppose we want to stabilize the period-2 orbit. We will implement parameter control when the system is near the period-2 point at $p=$ $0.8974359 \ldots$ The control function is given by $a(x)=f(p) / x(1-x)$, and the finite controlled system (parameterized over $M$ ) becomes $g_{M}(x)=f \circ F\left(a_{M}(x), x\right)$. Fig. 1 illustrates the resultant system. When we compare the graphs of $g_{M}$ for $M=20$ and 1000, we see that, as expected, the more accurate measurement yields a function with a smaller range in the vicinity of the fixed point, which produces a smaller invariant set. In each bin, all states are assigned the same measured value and therefore the same control signal, so $a_{M}$ is constant over each bin (Fig. 1c). The measurement and the control are exact at the center of each bin, so regardless of bin size, the center of each bin is mapped to the target point. The resultant controlled map, $g_{M}$, can be viewed as a piecewise collection of functions (Fig. 1d), selected from the family $f \circ F(a, x)$, parameterized over $a$. Within each bin, the appropriate family member is the function that maps the bin center to the fixed point.

The above example leads to our first generalization: To stabilize the system, the finite control system must operate with sufficiently small bins so that there is a trapping region that iterates to an invariant set containing the target point.

Proposition 1. Let $p$ be a fixed point of $f^{k}(x), F(a, x)$ be $C^{1}$ on some open region $I_{a} \times I_{x}$ containing $\left(a_{0}, p\right)$, and $a(x)$ be the continuous parameter control function, with $a\left[I_{x}\right] \subset I_{a}$. Then for any closed interval $I \subset I_{x}$ and any $\epsilon>0$, there exists an $M_{0}$ such that for all $M>M_{0},\left|g_{M}(x)-p\right|<\epsilon$ for all $x \in I$.

\footnotetext{
${ }^{1}$ A more general description of the measurement process is that we partition some interval $I$ into disjoint, half-open subintervals $I_{j}$, each of width $1 / M$. We call each subinterval a measuring bin (or simply bin). To each bin, we assign a measured state $x_{j}$ so that $x_{j} \in I_{j}$ and $x_{\mathrm{M}}(x)=x_{j} \forall x \in I_{j}$. The partition, together with the choice for each $x_{j}$, forms a bin scheme. Eq. (1) is a specific choice for a bin scheme.
} 
(a)

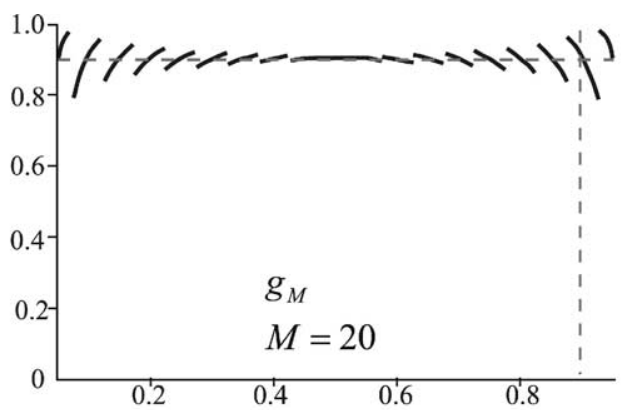

(c)

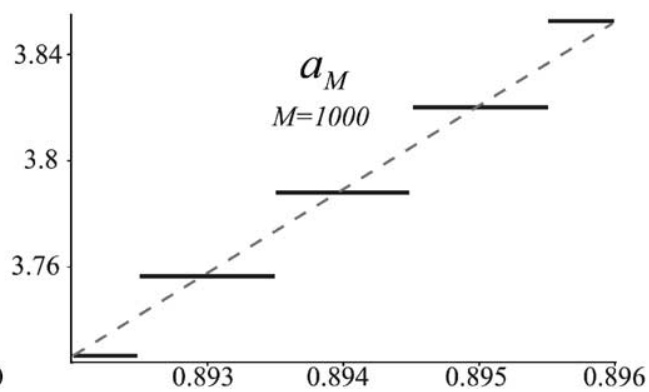

(b)

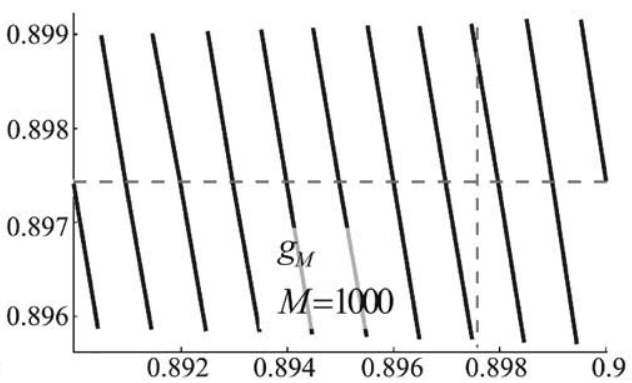

(d)

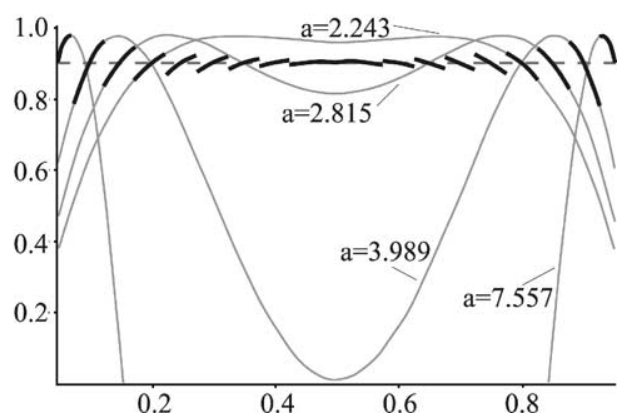

Fig. 1. Example of a finite controlled map: (a) $g_{M}$ for $M=20$ and (b) $M=1000$. The dashed line indicates the target point. (c) Calculated control (solid black). The dash line shows the exact control signal required. $a_{M}$ is constant over each bin and exact only in the middle of each bin. (d) $g_{M}$ is a piecewise collection of the family of functions $f \circ F(a, x)$, parameterized over $a$.

Simply stated, this proposition says that on a closed interval strictly inside the region where the exact control algorithm will work, there is some measurement precision that will allow the finite control algorithm to work to any required accuracy. To prove the proposition, we will bound $g_{M}^{\prime}$, which will limit the deviation from the fixed point over any bin.

Proof. Using the chain rule, ${ }^{2}$ from Eq. (2) we calculate $g_{M}^{\prime}$

$$
g_{M}^{\prime}(x)=\left.\frac{\mathrm{d}}{\mathrm{d} x}\left(f^{k-1}(x)\right)\right|_{F\left(a_{M}(x), x\right)}\left[\frac{\partial F}{\partial x}+\frac{\partial F}{\partial a} \frac{\mathrm{d} a}{\mathrm{~d} x_{\mathrm{M}}} \frac{\mathrm{d} x_{\mathrm{M}}}{\mathrm{d} x}\right] .
$$

Since $x_{M}$ is a step function, constant in each measuring bin,

$$
\frac{\mathrm{d} x_{\mathrm{M}}}{\mathrm{d} x}=0 \quad \text { a.e. } x \text {. }
$$

So

$$
g_{M}^{\prime}(x)=\left.\frac{\mathrm{d}}{\mathrm{d} x}\left(f^{k-1}(x)\right)\right|_{F\left(a_{M}(x), x\right)} \frac{\partial F\left(a_{M}, x\right)}{\partial x} .
$$

\footnotetext{
${ }^{2}$ As an alternative application of this development, if the discretization is in the control signal calculation or application of the control, a similar argument will still lead to the same mod map, Eq. (7). In either of those cases, $\mathrm{d} a / \mathrm{d} x=0$ a.e. $x$, and the rest of the argument follows analogously.
} 
To bound $g_{M}^{\prime}$ uniformly: Since $I_{x}$ is open, we can choose $M_{0}$ to make the bins small enough so that there is a closed set $U \subset I_{x}$ which contains all bins of size $1 / M_{0}$ that intersect the closed set $I$. Then for any $M>M_{0}$, $a_{M}[I] \subset a[U] \subset I_{a}$. Since $I$ and $a[U]$ are compact sets, we conclude

$$
\left|g_{M}^{\prime}\right|<\sup _{\substack{x \in I \\ a \in a[U]}}\left|\frac{\mathrm{d}}{\mathrm{d} x}\left(f^{k-1}(x)\right)\right|_{F(a, x)} \frac{\partial F(a, x)}{\partial x} \mid=K,
$$

where $K$ bounds uniformly in both $x$ and $M$.

In each bin, there is some point where the measured state is the same as the actual state $\left(x_{M}(x)=x\right)$. The control calculation will be exact and $g_{M}$ will map that point to $p$. Since the width of each bin is at most $1 / M_{0}$, the farthest the function can deviate from the fixed point is $K / M_{0}$. Choosing $M_{0}>K / \epsilon$ completes the proof.

The derivations above help us draw some additional conclusions. From Eq. (4), we see that if we accept a smaller desired trapping region $(I)$, then the value of $K$ may be reduced, and we may be able to achieve the required control precision without the need to measure as accurately (smaller $M_{0}$ ). Secondly, since we can choose $M$ to make the system operate on a "small" invariant set, a piecewise linear approximation (on the invariant set) may be appropriate. For $x$ near $p, x \approx p$ and $a_{M}(x) \approx a(p)=a_{0}$. From Eq. (3), we can conclude that if the invariant set is small, the slope of the map on that set is nearly constant and is approximated by

$$
\left.g_{M}^{\prime}(x) \approx \frac{\mathrm{d}}{\mathrm{d} x}\left(f^{k-1}(x)\right)\right|_{F\left(a_{0}, x\right)} \frac{\partial F\left(a_{0}, x\right)}{\partial x}=\frac{\mathrm{d}}{\mathrm{d} x}\left(f^{k}(p)\right) .
$$

\subsection{Approximating finite controlled systems by Renyí transformations}

For sufficiently large values of $M$, the map is well approximated by a mod map on the invariant set. Specifically, we can describe the linear approximation by

$$
g_{M}(x) \approx \tilde{g}_{M}(x)=p+\beta\left(x-x_{M}\right),
$$

where

$$
\beta=\frac{\mathrm{d}}{\mathrm{d} x}\left(f^{k}(p)\right)
$$

By a simple translation and scaling, the linear approximation (operating on the invariant set) is conjugate to a class of functions given by

$$
R(x)=R_{\alpha, \beta}(x)=(\alpha+\beta x) \bmod 1
$$

which maps $[0,1]$ to $[0,1]$, commonly called Renyí transformations. By referencing these maps of the unit interval, it will be easier to compare the characteristic of our controlled system for various values of the parameter $\beta$. In general, there is not a diffeomorphism between the original finite controlled system and the Renyí map, so any conclusions must be tempered by the realization that the kneading sequences and invariant densities of the actual system will be only finitely close to the linear approximation.

So far, we have shown that if we measure "accurately enough", the system will operate in a small invariant set about the desired fixed point, and on that invariant set, the map looks like a mod map, with slope on the invariant set approximately the same as the slope of the composed map at the fixed point. However, by focusing on the dynamics of the invariant set, we will draw much stronger conclusions. We can use the dynamics to allow us to have greater knowledge of the system or to increase the precision of our control algorithm. 


\section{Improved control techniques}

\subsection{Conventions and assumptions}

We assume the following:

1. In general, we will discuss techniques as if all maps are piecewise linear on the invariant set. We assume that a real system will be measured with sufficient accuracy that the linear approximation is reasonable.

2. After a short transient, the system will operate on a small invariant set containing the fixed point. We assume we are analyzing the system after this transient.

3. The techniques are most easily illustrated when the map $\tilde{g}_{M}$ has odd symmetry about the point $x=p, y=p$, formally stated as $\tilde{g}_{M}(p+x)-p=p-\tilde{g}_{M}(p-x)$. The related Renyí transformation, therefore, would be symmetric about the point $x=0.5, y=0.5 .^{3}$

Additionally, we will use the following conventions:

1. We will use a symbol dynamics to help us understand the techniques described. To each measuring bin in the invariant set of the original system, we assign a symbol (typically, $a, b$, or $c$ ). We associate the same symbol with the related region on the Renyí map. We refer to the interval as "bin $\gamma$ ". If the system state is in that bin, we say that the measured state is " $\gamma$ ".

2. The parameter $\beta$ is a characteristic of the original map and is not controlled. However, we will use ranges of that parameter value to characterize system behavior with the understanding that in comparing various values of $\beta$ we are actually comparing the behavior of different systems.

\subsection{Edge weighted maps: invariant density}

We investigate the discrete control system in the neighborhood of the target in terms of the linearization, Eq. (6), by considering the invariant probability density functions (pdfs) of the maps Eq. (7) with respect to the parameter $\beta$. For a given $\beta$, we examine the map created by placing the bins so that the fixed point is at the center of a bin. If $\beta$ is non-integer, these maps will have a small bin segment at each edge of the invariant set interval. We refer to this family of maps as edge-weighted maps. We analyze the time average asymptotic behavior of the controlled system by studying the invariant measures for maps from this family. The density function is easily and directly calculated in the case of rational $\beta$ by the dominant eigenvector of a Markov transition matrix [5,6] and is well approximated by a pdf of a nearby map of rational slope, in the case of irrational $\beta$ $[7] .{ }^{4}$

If $\beta$ is an integer, the invariant density is uniform. Otherwise, the invariant density generally trends toward more non-uniformity as the magnitude of slope gets smaller (see Fig. 2). For $|\beta|$ only slightly larger than integer, the small edge bins provide additional pre-images for the edges of the interval, so those bins carry significant weight in the associated density function. The higher-than-average density function over the outside bins (which exists for all non-integer values of $\beta$ ) is the genesis of our term edge-weighted maps. When $|\beta|<2$, the invariant

\footnotetext{
${ }^{3}$ Although symmetry of the map is not an a priori result of the general measuring scheme described in Section 2.2, we assume that the measuring bins can be adjusted either left or right, so that $p$ is at any desired position in the bin. Generally, we will choose $p$ to be at the center of the bin, although positioning $p$ at the edge of the bin also creates a symmetric map.

${ }^{4}$ In this work, we implement Ulam's method for approximating the Frobenius-Perron (FP) operator, and we iterate from an initial uniform density. For $|\beta|>\sqrt{2}$, the FP operator converges to its fixed point, the invariant density. For $|\beta|<\sqrt{2}$, the operator converges to an invariant density supported over only a periodic orbit, with the time average performance of the system estimated by averaging the densities over one period [6].
} 


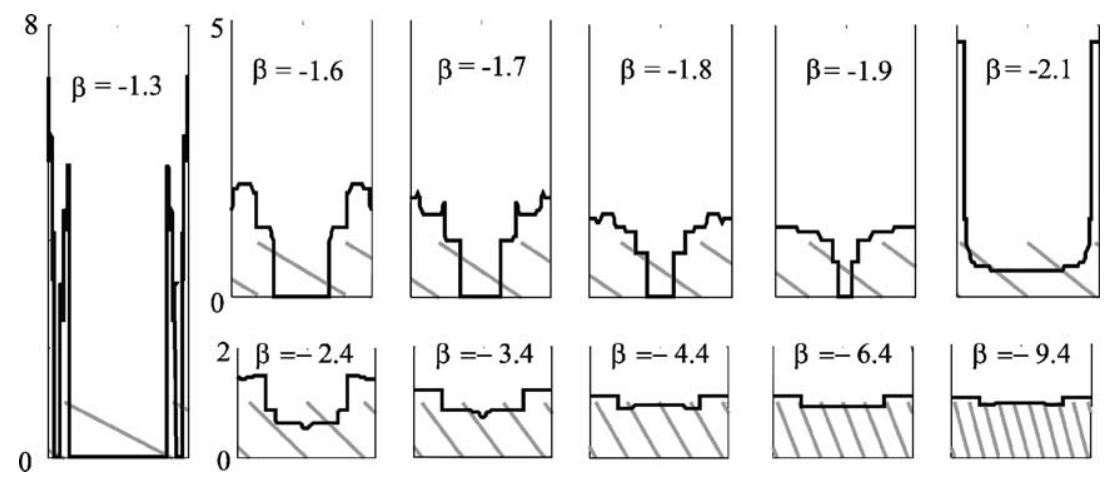

Fig. 2. Pdfs for edge-weighted maps. For each graph, the gray curve is the mod map that results from the control algorithm. The domain for each graph is limited to the interval of support for the invariant set, which is equivalent to the interval $[0,1]$ for the map of Eq. (7). The black curve is the invariant density associated with that map.

density is not supported over the entire interval, but has a "hole" in the middle. Orbits that start near the fixed point will be repelled from the fixed point until they enter one of the outer bins. Since the fixed point is not in the range of either of the outside bins, the orbit remains finitely far from the fixed point, with the size of the avoided region being $|\beta|-1 .^{5}$ The techniques we will describe in this paper exploit the non-uniformity of the densities of these maps. We focus our discussion on these small slope $(|\beta|<2)$ maps, where the non-uniformity is most pronounced.

\subsection{Edge-weighted maps: increased knowledge from kneading sequences}

The system operates finitely far from the fixed point (the target), so continued control action will be required. But the center bin has regions of non-zero density and is, therefore, always revisited. On each visit to the center bin, the measurement indicates the system is at the target, which would imply that no further control action is required. This "discrepancy", which could be inferred from the measurements, helps to underline the fact that the symbol dynamics generated by the partition from the measurement bins are not conjugate to the full dynamics of the map Eq. (7) [8]. We can take advantage of the difference between the measurement partition and a generating partition, using the past history of the orbit to determine the state of the system more accurately than is given by the measurement system.

Fig. 3 provides a means for us to visualize the interplay of the kneading sequences and the symbol dynamics. Although $g_{M}$ is defined on some large interval, only three measuring bins intersect the invariant set, and only the middle bin is completely inside the invariant set. We use a cobweb representation to understand the interval arithmetic that generates various symbol sequences.

Consider the symbol sequence $a b$. When $b$ is measured, we know that the system is not allowed to be anywhere in bin $b$, but is restricted to the interval whose pre-image is in that part of bin $a$ which is inside the invariant set. Similarly, when we measure $c$ following an $a b$, we know that the system is actually in the subset of $c$ whose pre-image is in interval $a b$. In both cases, we can estimate the state more precisely than the $1 / M$ bin width capability of our measuring system. For a given value of $\beta$, one can compute the intervals associated with each word by first computing the kneading sequence of $0[9,10]$.

\footnotetext{
${ }^{5}$ If $|\beta|<\sqrt{2}$, an analysis of the kneading sequence reveals that another avoided region develops in each of the outer bins. These intervals of non-support continue to emerge as $|\beta| \rightarrow 1$.
} 


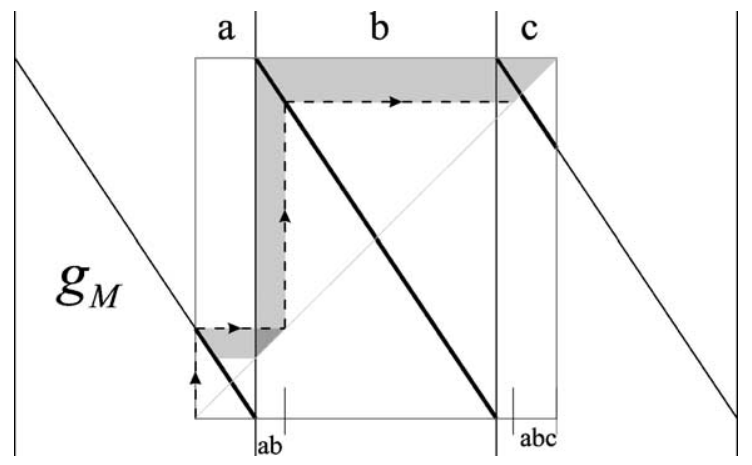

Fig. 3. Kneading sequences of edge weighted map, $\beta=-1.5 . g_{M}$ is shown over three measuring bins, with the gray box enclosing the invariant set. The solid curve is $g_{M}$. The dashed line shows the kneading sequence of 0 , which generates the intervals associated with $a b$ and $a b c$.

Table 1 is provided to illustrate two main points from this section:

1. The gain in knowledge of state can be quite significant. The smaller the magnitude of the slope, the more accurately the system can be known. Note that for $\beta=-1.3$, there are words for which the state is known to an accuracy more than 100 times better than is measured.

2. The piecewise linear map provides a reasonable approximation while using this technique to improve our knowledge of the state. The third column shows interval calculations for $f(x)=3.6 x(1-x)$ with $M=1000$. The slope of this map is -1.6 at the fixed point. The results are well approximated by the piecewise linear approximations for $\beta=-1.6$. If the control map is not computable in closed form, computation based on linearization is the only alternative. However, since only finite length words are generally relevant, the difference between the actual system and the piecewise linear system will be small.

Fig. 4 provides a graphical representation of the application of this technique to a dynamical system. The specific example uses a Renyí transformations with $\beta=-1.3$. The controlled system was used to generate the symbol sequence (measurements) shown. At each iteration, the vertical width of each mark represents the accuracy of the knowledge of the actual state of the system. For the upper graph, the vertical width is equivalent to one bin width, the nominal accuracy of our measuring system. From that same symbol stream, our analysis uses kneading sequences

Table 1

Calculated accuracies from kneading sequences ${ }^{\mathrm{a}}$

\begin{tabular}{|c|c|c|c|c|c|}
\hline \multicolumn{2}{|c|}{$\beta=-1.3$} & \multicolumn{2}{|c|}{$\beta=-1.6$} & \multicolumn{2}{|c|}{$f(x)=3.6 x(1-x)$} \\
\hline Word & Size & Word & Size & Word & Size \\
\hline$a, c$ & 0.15 & $a, c$ & 0.3 & $a$ & 0.3009 \\
\hline$a b, c b$ & 0.045 & & & $c$ & 0.3045 \\
\hline$a b c$ & 0.0585 & $a b, c b$ & 0.18 & $a b$ & 0.1834 \\
\hline$a b c c$ & 0.03105 & & & $c b$ & 0.1852 \\
\hline$a b c c c$ & 0.04365 & $a b c$ & 0.288 & $a b c$ & 0.2983 \\
\hline$a b c c c c$ & 0.007575 & & & $c b a$ & 0.2969 \\
\hline$a b c c c c c$ & 0.009717 & $a b c c$ & 0.2808 & $a b c c$ & 0.2946 \\
\hline & & & & cbaa & 0.2891 \\
\hline
\end{tabular}

a Interval size for various words, given in fraction of a measuring bin. The first two columns are Renyí maps. The third column shows calculations for the logistic map, with $f(x)=3.6 x(1-x)$ and $M=1000$ (the slope is -1.6 at the fixed point) to illustrate the reasonableness of using the piecewise linear approximation to perform calculations. 


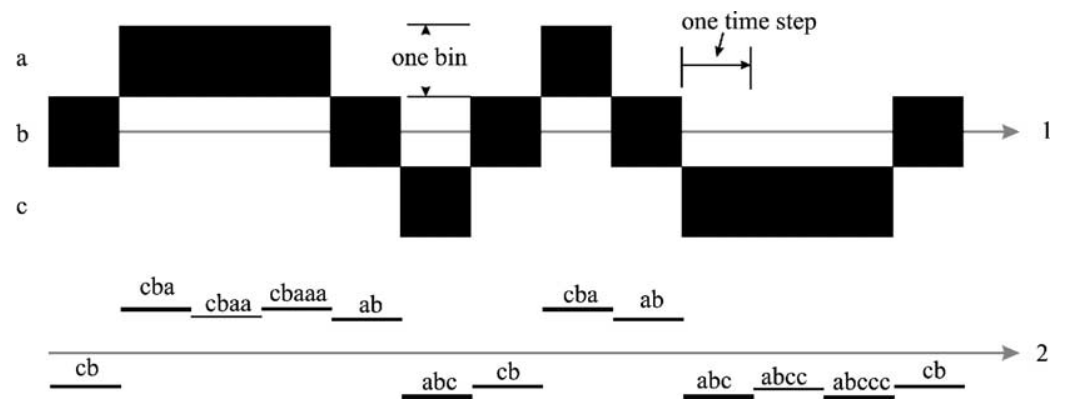

Fig. 4. Improving knowledge of state in 1D. Signal 1 graphically represents the measured state of a controlled system. The height of each block represents the interval of possible states associated with that measurement. Signal 2 shows the same iterations of the system. The vertical height gives the interval for the state based on the symbol sequence labeled above each iteration.

and interval arithmetic to produce the second line of data. The two signals are plotted with the same vertical scaling, with the vertical width of the signal at each iteration being proportional to the size of the interval associated with that symbol sequence. In Signal 2, each iterate is labeled with the symbol sequence history. That history is used to calculate the known interval for the state of the system, based on the techniques described above. Table 1, therefore, gives the width of Signal 2 as a fraction of the bin width established by Signal 1.

\subsection{Improved control accuracy algorithm}

The average density associated with an edge-weighted map $(|\beta|<2)$ has a "hole" around the targeted point (see Fig. 2). If the bin placement is shifted so that the target is at the edge of a bin, the map has two pre-images for the center of the invariant set. We call this system center-weighted. Edge-weighted bin placement allows more accurate knowledge of system state. But if the goal is to keep the system as close as possible to the target point (on average), then the center-weighted bin placement would be more appropriate. However, a better way to improve the average control accuracy is to modify the edge map as shown in Fig. 5. We call this map the improved-accuracy map. The bin placement remains the same as in the edge-weighted map. However, in bins $a$ and $c$, the map is shifted vertically so that the invariant set portion of those legs is centered on the target point. The map provides three pre-images for the target (versus two for center-weighted maps).

Recall that one way to describe the original algorithm is that the control scheme selects a different value of the control parameter for each bin, creating a piecewise function with the center of each bin mapped to the target point. Since the target point is at the center of bin $b$, the control parameter is $a_{0}$, the parameter value of the original map;

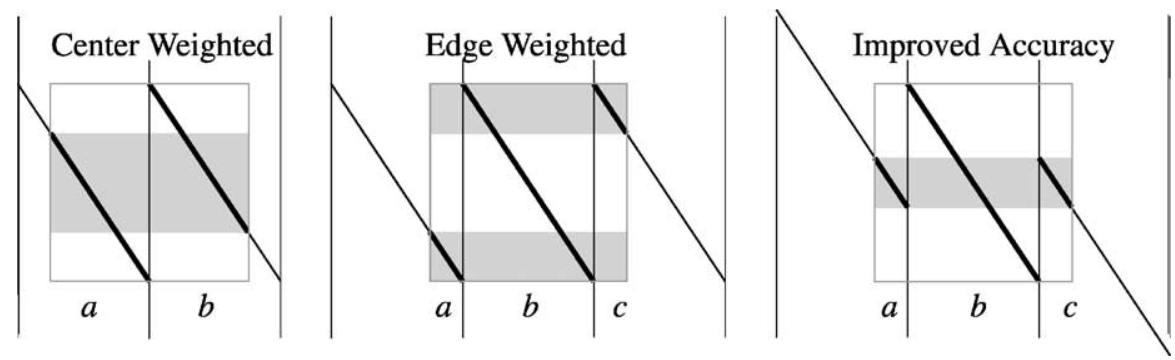

Fig. 5. Targeting the center of the invariant set. The improved-accuracy map has the same bin setup as the edge-weighted map, but the map is shifted vertically in the edge bins. It provides three pre-images of the target point. 

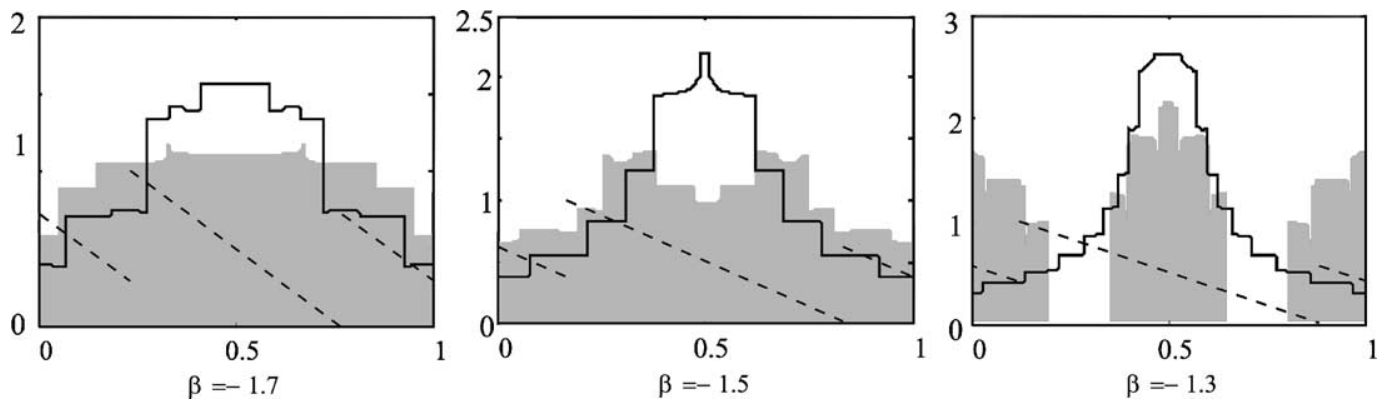

Fig. 6. Improved algorithm performance. For each graph, the gray area graphs the average density for the center weighted and the solid curve shows the performance of the improved accuracy method. For each, the improved accuracy map is also shown as the dashed curve.

bins $a$ and $c$ require slightly different parameters:

$$
a(x)= \begin{cases}a_{0}+\delta^{+} & x \in \operatorname{bin} a, \\ a_{0} & x \in \operatorname{bin} b, \\ a_{0}+\delta^{-} & x \in \operatorname{bin} c .\end{cases}
$$

If a linear approximation is accurate, then $\delta^{+} \approx-\delta^{-}$. In essence, the algorithm may be accurately implemented on the invariant set with only one control signal generator, the perturbation delta. The control response is a 0 input if the measured state is $b$, and $\pm \delta$ for measured states $a$ and $c$.

The improved-accuracy map requires an alteration to the basic control algorithm, but the new algorithm can be executed without adding complexity. We simply calculate the required parameter perturbation $\hat{\delta}$ to select the member of family of parameter curves that centers the invariant set portion of bin $a$ on the target point (refer to Fig. 1). Under the linearization assumption $\hat{\delta}^{+} \approx-\hat{\delta}^{-}$, the revised control algorithm on the invariant set operates exactly as above, selecting either perturbation 0 or perturbation $\pm \hat{\delta}$ :

$$
\hat{a}(x)= \begin{cases}a_{0}+\hat{\delta}^{+} & x \in \operatorname{bin} a, \\ a_{0} & x \in \operatorname{bin} b, \\ a_{0}+\hat{\delta}^{-} & x \in \operatorname{bin} c .\end{cases}
$$

Since the determination of the revised perturbation parameters is done during the development of the algorithm, the improved system requires no additional real-time calculations to implement, but performance is significantly improved in the sense that the pdf for that system is more highly peaked near the target point. Fig. 6 shows a comparison of the density for the center-weighted map (gray area graph) to the improved-accuracy density (solid line) for three different values of $\beta .{ }^{6}$ In addition to the overall reduction in variance, the improved density decreases monotonically with distance from the target point.

\section{Numerical application to stabilization of saddles in $R^{2}$}

When we study finite accuracy feedback control systems in $R^{2}$, we see that the basic ideas from the 1D case still apply:

- Dynamics on the invariant set are well described by a piecewise linear approximation;

${ }^{6}$ Densities are invariant, except for the slope -1.3 center-weighted map, where the bimodal nature of the center-weighted graph (whose slope is $<\sqrt{2}$ ) forces the use of an average density over one period. 
- We can achieve better control by assigning a specific control signal for each of the finite measurements instead of using a single (global) rule that calculates control based on the measured state (the center of each measuring bin);

- By analyzing symbol sequences on the finite set of measurements in the invariant set, we can know the state of the system with greater accuracy than measured.

However, where the 1D case allowed us to describe characteristics of these maps over the parameter space described by $\beta$, we have not yet found a "nice" parameter space to characterize the $2 \mathrm{D}$ case. The analysis techniques can achieve numerically significant improvements in performance but require study of a specific system and algorithm. As such, we limit ourselves to a general description of how the analysis would be applied to the OGY [1] algorithm for stabilizing a saddle fixed point, and provide a specific example by analyzing finite accuracy measurement control of the Ikeda map.

\subsection{OGY technique under finite accuracy measurements}

Consider the map,

$$
\begin{aligned}
& \mathbf{x}_{n+1}=\mathbf{f}\left(\mathbf{x}_{n}\right), \\
& \mathbf{f}\left(\mathbf{x}_{n}\right)=\mathbf{F}\left(\mathbf{x}_{n}, p_{0}\right),
\end{aligned}
$$

where $\mathbf{x}_{n} \in \mathbf{R}^{2}, \mathbf{F}\left(\mathbf{x}_{n}, p\right)$ is a smooth function, and $p$ a scalar control parameter, with nominal value $p_{0}$. Let $\mathbf{x}_{F}\left(p_{0}\right)$ be a saddle fixed point of (10). The OGY [1,11-13] algorithm is a state feedback control scheme that provides the necessary calculations to determine the required control signal $p$ to stabilize the fixed point when the state is within an $\epsilon$ neighborhood of $\mathbf{x}_{F}\left(p_{0}\right)$. Since OGY is well described in other references [1], we review OGY in only brief details.

Assume that for any $\mathbf{x}$, the required control is given by $p(\mathbf{x})$ such that

$$
\mathbf{x}_{n+1}=\mathbf{F}\left(\mathbf{x}_{n}, p\left(\mathbf{x}_{n}\right)\right) \in E_{\mathrm{s}}\left(\mathbf{x}_{F}\left(p_{0}\right)\right) .
$$

Near the fixed point, $E_{\mathrm{s}}$ is near the stable manifold $\left(W_{\mathrm{s}}\right)$ of the fixed point, so unperturbed iterations of the map would move the orbit generally toward the fixed point. If $W_{\mathrm{s}}$ is non-linear, further parameter perturbations will be required to maintain the system near the fixed point. Now assume that the state is not known exactly. Rather, each coordinate of the state is measured using a finite accuracy system as described in Section 2.2, with the measured state given by the vector

$$
\mathbf{x}_{\mathrm{M}}=\left(\begin{array}{l}
x_{\mathrm{M}_{1}}\left(x_{1}\right) \\
x_{\mathrm{M}_{2}}\left(x_{2}\right)
\end{array}\right) .
$$

The control perturbation is calculated as $p_{M}(\mathbf{x}) \equiv p\left(\mathbf{x}_{\mathrm{M}}\right)$. Control is active on each iteration to yield the control map

$$
\mathbf{g}_{M}(\mathbf{x})=\mathbf{F}\left(\mathbf{x}, p\left(\mathbf{x}_{M}\right)\right) .
$$

The geometric interpretation of this procedure is that the measuring scheme partitions $R^{2}$ into rectangles of size $1 / M_{1} \times 1 / M_{2}$. A single vector value (the measured state) is assigned to each rectangle, and the system is controlled so that the measured state maps onto the $E_{\mathrm{s}}$. Since control is activated only when the system is near the fixed point, linearization is appropriate, and the image of a rectangle is approximately a parallelogram centered about the point that is the image of $\mathbf{x}_{\mathrm{M}}$ under the standard OGY algorithm. Under suitable conditions for successful OGY execution 
(in the exact measurement case), the system with finite accuracy can be measured with sufficient precision to achieve an attracting invariant set in the vicinity of the fixed point.

In the $1 \mathrm{D}$ case, we gave general descriptions by parameterizing over $\beta$ (the slope at the fixed point). Similarly, the stability of variations of the 2D maps are characterized by the Jacobian derivative matrix, evaluated at the fixed point

$$
\mathbf{J}=\left.\frac{\mathrm{d} \mathbf{F}}{\mathrm{d} \mathbf{x}}\right|_{\mathbf{x}_{F}\left(p_{0}\right)} .
$$

However, because the measurement grid from Eq. (12) is generally not aligned with the eigenvectors associated with $\mathbf{J}$, a linear change of coordinates to the eigenvector basis does not simplify the description of the piecewise function. Additionally, the size and character of the 2D maps are highly dependent upon the direction the fixed point is moved in response to control perturbation, as described by $\partial \mathbf{x}_{F} /\left.\partial p\right|_{p=p_{0}}$ to first-order. As such, for a given map and fixed point, although $\mathbf{J}$ is unchanged, the finite controlled map will depend upon the specific choice of which parameter is used as the control parameter. Therefore, five parameters are required to completely discuss this family of finite control maps (in 2D). Because we were interested in the specific aspects of "improving" the control algorithm, we have not pursued general conclusions. Instead, we use numerical simulations to illustrate how analysis of a specific problem yields worthwhile results in both

1. providing a means for better control,

2. increasing knowledge of the state.

\subsection{Ikeda map: a case study of the $2 D$ problem}

Consider the Ikeda map, given by

$$
F(x, y, p)=\left(\begin{array}{c}
(1+p)-0.9(x \cos \tau+y \sin \tau) \\
0.9(x \sin \tau+y \cos \tau)
\end{array}\right)
$$

with $\tau=0.4-6 /\left(1+x^{2}+y^{2}\right)$ and nominal parameter $p_{0}=0$. The map has a saddle fixed point at (1.054972676, -2.174591983 ) with eigenvalues of $\lambda_{u}=-1.221$ and $\lambda_{s}=0.6634$. We implement OGY [1] control based on measuring the system under an $x y$ grid with $M_{1}=M_{2}=1000$. The measurement grid is placed so that the fixed point is at the center of a measurement box, and in each box, the assigned measured state is the center of the box. A large set of initial conditions are placed randomly near the fixed point. Under repeated application of the controlled map, the points appear to converge to a chaotic attractor. The resultant graph is shown in Fig. 7. After several iterations, the mean variance (from the fixed point) changes little under iteration, and we assume that the map is converging to an invariant density which is supported over a Cantor set.

We choose the mean variance from the fixed point $v$ as both our evaluation function of the control algorithm as well a means to compare the model and its linear approximation. For the Ikeda map with parameters as chosen, $v \approx 0.609 \times 10^{-3}$, while the linearized model has variance $\tilde{v} \approx 0.607$. The "closeness" of the linear approximation (when corrected for scaling) indicates that the analysis may be performed even when a closed form description of the non-linear map is not available, as long as the eigenvalues and eigenvectors of the Jacobian can be determined.

\subsection{Improving the $2 D$ algorithm}

The general effect of finite accuracy measurement is that all states inside a specific measuring box are assigned the same measured state $\left(x_{\mathrm{M}}, y_{\mathrm{M}}\right)$, and therefore the associated control parameter must be the same for all states 


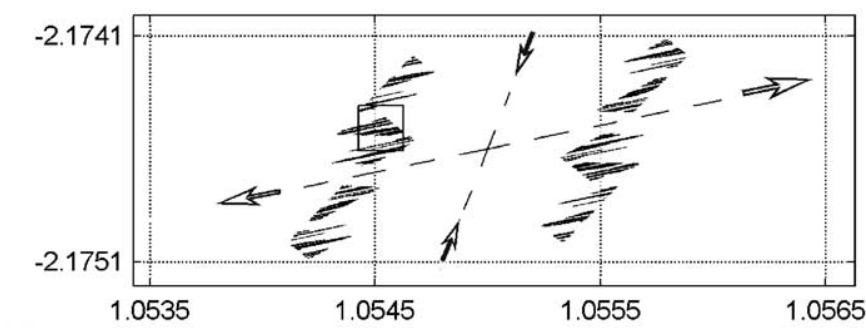

(a) (b)

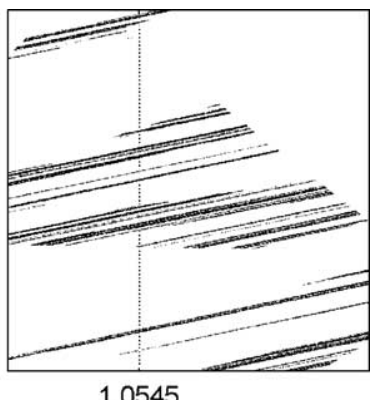

1.0545

Fig. 7. Finite accuracy control in $R^{2}$. (a) Numerical approximation of the invariant set of the Ikeda map stabilized via OGY with state measurements to the nearest $1 / 1000$. The dashed lines and arrows show the stable and unstable subspaces. (b) Zoomed to the boxed area of (a) to better illustrate that the controlled system appears to operate on a Cantor set.

inside that box. Our goal, therefore, is to find the "best" control parameter perturbation to associate with each box. The geometric interpretation would be that instead of mapping the center of each box onto $E_{\mathrm{s}}$ (as described in Section 4.1), some point other than the center of the box is mapped to $E_{\mathrm{s}}$. We call that point the optimal control point for the box. Since we are concerned only with the behavior of the invariant set (which is bounded), we need to find only a finite set of optimizing parameter values or optimal control points.

If we focus on the linearization, we note that the map will be symmetric, so the invariant set will occupy an odd number of boxes. If the invariant set occupies $2 k+1$ boxes, then a general optimization is over $2 k+1$ parameter values. However, if the cost function is even, the box containing the fixed point can be assigned control parameter $p=0$, and a symmetry argument tells us that the optimization is over $k$ control parameter values. Solving this optimization problem with a generalized cost function is made difficult by the fact that the cost must be evaluated over the invariant set, which we believe to be fractal in nature and not computable in closed form. This paper does not try to address efficient algorithms for solving the optimization problem, but merely asserts that parameters other than those from a straightforward OGY calculation may provide improvement.

As a specific example of improved control, we address the Ikeda map problem from Section 4.2 with the same cost function of mean variance from the fixed point. Since the invariant set covered only three boxes, our goal was to optimize over a single parameter. We used a relatively unsophisticated approach of approximating the invariant set via Monte Carlo simulation and conducted a line search using a bisection method with several seed values. Our search yielded a parameter value that was equivalent to a control point of $\left(x_{M}-(0.509 / M), y_{M}\right)$ for the right box and the symmetric point for the left. As before, we iterate a large ensemble of random initial conditions until the average variance stabilizes and we assume that we have approximated the invariant density. The average variance over that set is $v \approx 0.193 \times 10^{-3}$ (compared to $0.609 \times 10^{-3}$ for the standard OGY control of Section 4.2). Fig. 8 shows the numerical approximation of the resultant invariant set. The asterisks in the figure plot the optimal control point for the left and right boxes. As in the case with standard algorithm, the system appears to live on a Cantor set.

\subsection{Increased knowledge of state in a $2 D$ system}

In the 1D model, improving the accuracy of system control and increasing the knowledge of the actual state were considered as an analysis of two different maps. If we consider "state knowledge" as the area associated with a given measurement (a single symbol), then simply determining the invariant set provides improvement. If the 

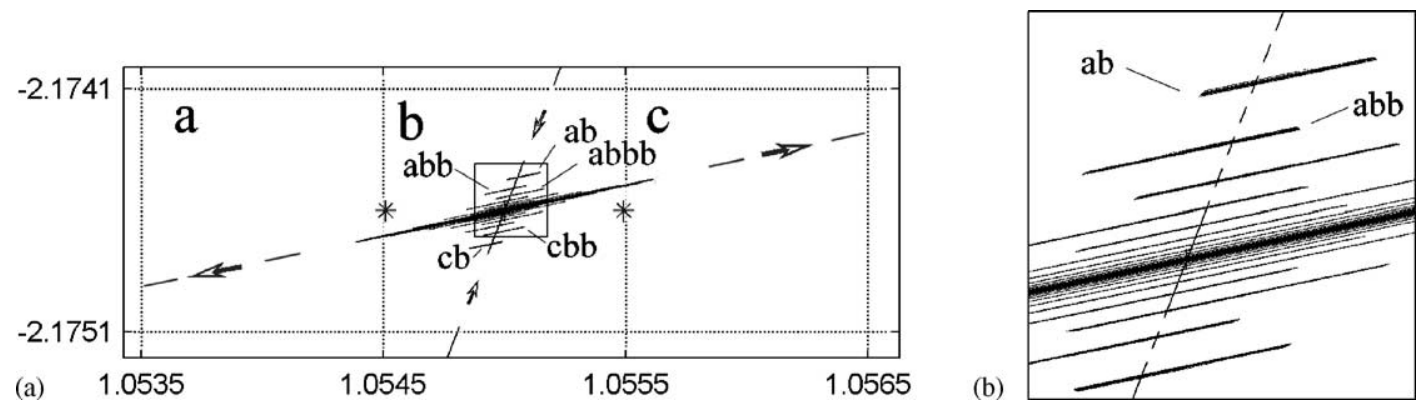

Fig. 8. Improved control in $R^{2}$. (a) To improve control of our Ikeda map, we used control points marked by asterisks for the left and right measurement bins. We iterate a large ensemble of initial conditions to estimate the invariant set. The dash and arrow lines show the stable and unstable manifolds for the map. (b) A zoomed copy of the boxed area in (a). We associate each measuring box with a symbol (a, b, or c), and label various portions of the invariant set with the associated symbol sequence.

determinant of the Jacobian has magnitude less than one (as for this Ikeda map), then the contraction under the map must result in a measure-zero set in $R^{2}$. Although we cannot determine the invariant set exactly, we can find a small set that covers the invariant set and use it to approximate the state for a given symbol. In the 2D case, the added dimensionality allows us to optimize some global function while still (possibly) achieving higher resolution of state with the symbol dynamics. Fig. 8 is annotated to indicate the symbol sequences associated with some of the "striations" of the invariant set of our Ikeda map, where the parameters have already been manipulated to optimize the global performance. If we associate a symbol with each measurement box, then we can associate a finite symbol sequence with various portions of the invariant set. If the set is a Cantor set (as appears from simulations) then improved estimates of state would be based on some small set that covers the appropriate portion of the invariant set. The figure provides an easy means of visualizing the improved knowledge of state, since each measurement (a symbol) would be a $0.001 \times 0.001$ square in $R^{2}$. It appears as though the mapping is transitive, so eventually, even a small subset of initial conditions will get stretch over the whole set. However, we can track a symbol sequence for several iterations (and gain improved measurement) before we lose resolution in the invariant set. Because the Ikeda map is area contracting, each iteration indicates an improvement in knowledge. However, our experimentation with other systems indicates that even for expanding maps, analysis of even short (1-3 symbols) sequences yields significant gains in knowledge of state variables.

\section{Conclusion}

The foundation of many feedback control systems is some process that converts from analog to digital. Typically, systems have been designed so that the inaccuracies of that approximation are within the tolerances of the system, so little attention is given to the dynamical system that is actually in place. However, an analysis of that system may yield significant improvements, both in the sense of state observation as well as time average performance. In the 1D case, the resultant dynamics are easily described, in a general sense, by the fact that the controlled system generates a simple mod map structure whose slope is given by the slope of the original unstable map at the fixed point. In higher dimensions, general analysis becomes more difficult, but analysis of specific control problems may prove very useful, with improvement in performance and greater resolution simultaneously achievable. For the higher dimensional problem, this paper considered only saddle points stabilized with the OGY algorithm. However, the basic idea of analyzing the dynamics on the invariant set of the deterministic system should prove useful, regardless of the particular circumstance of instability and choice of control algorithm. 


\section{Acknowledgements}

EMB was supported by the NSF under Grant No. DMS-0071314.

\section{References}

[1] E. Ott, C. Grebogi, J. Yorke, Controlling chaos, Phys. Rev. Lett. 63 (11) (1990) 1196-1199.

[2] T. Shinbrot, C. Grebogi, E. Ott, J.A. Yorke, Using chaos to direct trajectories to targets in systems describable by a one-dimensional map, Phys. Rev. A 45 (1992) 4165-4168.

[3] H. Kantz, T. Schriber, Nonlinear Time Series Analysis, Cambridge University Press, New York, 1997.

[4] J.D. Farmer, J.J. Sidorowich, Optimal shadowing and noise reductions, Physica D 7 (1993) 153.

[5] P. Góra, A. Boyarsky, Laws of Chaos, Invariant Measures and Dynamical Systems in One Dimension, Birkhäuser, Boston, 1997.

[6] A. Lasota, M. Mackey, Chaos, Fractals, and Noise, 2nd ed., Springer, New York, NY, 1997.

[7] L. Billings, E. Bollt, Probability density functions of some skew tent maps, Chaos Solitons Fract. 12 (2001) 365-376.

[8] E. Bollt, T. Stanford, Y.-C. Lai, K. Zyczkowski, What symbolic dynamics do we get with a misplaced partition? On the validity of threshold crossings analysis of chaotic time-series, Physica D 154 (3-4) (2001) 259-286.

[9] J. Milnor, W. Thurston, On Iterated Maps of the Intervals I and II, Princeton University Press, Princeton, 1977;

J. Milnor, W. Thurston, On Iterated Maps of the Interval, Dynamical Systems, Lecture Notes in Mathematics, vol. 1342, Springer, Berlin, 1988, pp. 465-563.

[10] W. de Melo, S. van Strien, One-dimensional Dynamics, Springer, Berlin, 1993.

[11] F. Romerias, C. Grebogi, E. Ott, W. Dayawansa, Contolling chaotic dynamical systems, Physica D 58 (1002) (1992) 165-192.

[12] G. Chen, X. Dong, From Chaos to Order Methodologies, Perspectives and Applications, World Scientific, Singapore, 1997.

[13] H.-G. Schuster (Ed.), Handbook of Chaos Control: Foundations and Applications, Wiley, New York, 1999. 